\title{
Electrically conducting polymer nanostructures confined in anodized aluminum oxide templates (AAO)
}

\author{
I. Blaszczyk-Lezak, V. Desmaret, C. Mijangos* \\ Instituto de Ciencia y Tecnología de Polímeros, CSIC, Juan de la Cierva 3, 28006 Madrid, Spain
}

Received 16 July 2015; accepted in revised form 15 October 2015

\begin{abstract}
Intrinsically or extrinsically conducting polymers are considered good candidates for replacement of metals in specific applications. In order to further expand their applications, it seems necessary to examine the influence of confinement effects on the electric properties of nanostructured conducting polymers in comparison to the bulk. The present study reports a novel way to fabricate and characterize high quality and controllable one-dimensional (1D) polymer nanostructures with promising electrical properties, with the aid of two examples polyaniline (PANI) and poly(vinylidene fluoride) with multiwall carbon nanotubes (PVDF-MWCNT) as representative of intrinsically and extrinsically conducting polymers, respectively. In this work, porous anodic aluminum oxide (AAO) templates have been used both as a nanoreactor to synthesize 1D PANI nanostructures by polymerization of the ANI monomer and as a nanomold to prepare 1D PVDFMWCNT nanorods by melt infiltration of the precursor PVDF-MWCNT film. The obtained polymer nanostructures were morphologically and chemically characterized by SEM and Confocal Raman Spectroscopy, respectively, and the electrical properties determined by Broadband Dielectric Spectroscopy (BDS) in a non-destructive way. SEM study allowed to establish the final nanostructure of PANI and PVDF-MWCNT and confirmed, in both cases, the well-aligned and uniform rodlike polymer nanostructures. Confocal Raman Microscopy has been performed to study the formation of the conducting emeraldine salt of PANI through all the length of AAO nanocavities. Finally, the electrical conductivity of both types of polymer nanostructures was easily evaluated by means of Dielectric Spectroscopy.
\end{abstract}

Keywords: nanocomposites, conducting polymers, Broadband dielectric spectroscopy, AAO template

\section{Introduction}

Conducting polymers are considered good candidates to replace traditional metals in specific applications. Indeed, this new generation combines advantages of polymers (lighter weight, mechanical flexibility, easy-processing, biocompatibility, chemical stability) and conducting materials (electrical properties). Among polymers, conduction can be achieved by the intrinsically conducting polymers (ICP), i.e., polymers with alternating double bonds in their chemical structure or by the extrinsically conducting polymers (ECP), i.e., polymers with added conductive nanofillers to the matrix.

\footnotetext{
${ }^{*}$ Corresponding author, e-mail: cmijangos@ictp.csic.es

(C) BME-PT
}

In order to further improve the properties of these materials, as well as expand their applications, the study of the polymer nanostructures could have some advantages, like in case of one-dimensional polymer nanostructures (1D). In fact, it was reported that nanofibrils exhibit higher electronic conductivities compared to conventional forms of the same polymer material [1]. It has been also reported potential applications of nanostructured conducting polymers that include electromagnetic $[2,3]$ and radio frequency interference shielding for electronic devices, electrostatic charge dissipation [4] and conductive paints [5], organic light emitting diodes (OLED) [6], bio- [7] and chemical sensors [8,9], photovoltaics 
$[10,11]$, electrochromic devices [12], energy storage $[13,14]$, corrosion protection [15], plastic digital memory [16], bio-engineering [17] etc.

One of the routes to synthesize one-dimensional conducting polymer nanostructures is the use of anodized aluminum (AAO) hard templates since dimension, aspect ratio and electrical properties of the resulting polymer nanostructures can be tailored and therefore it can provide new opportunities for confinement studies and/or for technological challenges. In this sense, a few years ago, Martin and coworkers [18, 19], reported that the polymerization of ANI into the pores of polycarbonate filters improved the conducting properties of the polymer in comparison to bulk. He suggested that the improvement in electronic conductivity results from enhancement in the supermolecular order within the template-synthesized fibers. Authors used four-point conductivity measurement which requires the preparation of thin films. Some years later, Marquez and coworkers [20, 21], presented a different approach to this topic. The author synthesized patterned polymeric nanostructures by template assisted admicellar polymerization (TAAP) and studied AC impedance measurements (via a current-sensing AFM apparatus). The results revealed a dependence of electrical conductivity values of PANI honeycombs not only on polymerization conditions and doping but on the template size too (biggest spheres, biggest conductivity). Recently, Wu et al. [22] investigated the conductivity of doped with different acids PANI nanoarrays prepared through a soft template method. The thermoconductivity measurements showed an organic semiconductor characteristic, so that, the electrical conductivity increase with the rise of temperature. Another way of preparing PANI nanostructures was presented by Khdary et al. [23]. Authors produced high surface area mesoporous polyaniline on the surface of glassy carbon electrodes by the electrochemical polymerization from a composite obtaining high value of capacitance.

To our best knowledge, although Broadband dielectric spectroscopy (BDS) is a sensitive technique to evaluate dielectric response of polymer nanostructure [24-28] and to measure polymer conductivity as a function of frequency and temperature, it has never been employed to study the electrical response of confined conducting polymers in AAO templates, even if this type of measurement have been currently done in bulk and thin films [29]. In addition, it is possible to use the template without additional preparation. The aim of this work is to evaluate the electric conducting properties of two kind of conducting polymer confined in the nanocavities of AAO templates. Polyaniline (PANI) has been chosen as representative of an intrinsically conducting polymer (ICP) and PVDF-MWCNT composite, made of poly(vinylidene fluoride) (PVDF) and multiwall carbon nanotubes (MWCNT), as representative of the extrinsically conducting polymer (ECP). Laboratory-made anodized aluminium oxide templates with controlled pore sizes have been employed for polymer nanostructures preparation. Polyaniline nanostructures have been obtained by 'in situ' polymerization of ANI in the AAO nanopores while PVDF-MWCNTs nanorods, by melt infiltration of a PVDF-MWCNTs film in the AAO nanopores. Their posterior morphological, chemical and electrical characterization has been carried out by SEM, Raman and BD spectroscopy.

\section{Experimental \\ 2.1. Materials}

Aluminium foils of $99.999 \%$ were purchased from Goodfellow Cambridge Ltd. (Huntingdon, United Kingdom); Anilyne ( $\geq 99.5 \%)$ and PVDF $\left(M_{\mathrm{w}} \approx\right.$ 180000) supplied by Sigma-Aldrich (Madrid, Spain); Anhydrous N,N-dimethylformamide (DMF) provided by Carlo Erba Reagents (Sabadell, Spain) (purity $\sim 99.9 \%$ ); and MWCNTs synthesized by the M.A. Lopez Manchado (Instituto de Ciencia y Tecnología de Polímeros, CSIC, Madrid, Spain) (nominal inner diameter $d_{\mathrm{i}} \sim 12.5 \mathrm{~nm}$, outer diameter $d_{0} \sim$ $44 \mathrm{~nm}$ and length $1 \sim 160 \mu \mathrm{m})$.

\subsection{AAO template synthesis}

Two types of ordered AAO templates have been prepared by a two-step electrochemical anodization process of aluminum foils. Firstly, ultrapure aluminum foils were cleaned and degreased by sonication in solvents of different polarity (acetone, isopropanol, deionized water and ethanol). Then, they were electropolished during $4 \mathrm{~min}$ in a solution of perchloric acid/ethanol (1/3) under a constant voltage of $20 \mathrm{~V}$ with a maximum current of $1.5 \mathrm{~A}$. After that, in order to prepare templates with $35 \mathrm{~nm}$ diameter of pores, the first anodization was achieved using oxalic acid as electrolyte at $40 \mathrm{~V}$ and $3-5^{\circ} \mathrm{C}$ for $24 \mathrm{~h}$ in a subsequent step, the anodic layer was removed into chromic and phosphoric acid solution. This was 
followed by a second anodization process also in oxalic acid under the same conditions but during $65 \mathrm{~h}$. After this stage, templates are characterized by an anodic aluminum oxide top layer with pores of $35 \mathrm{~nm}$ in diameter arranged into a hexagonal lattice of $100 \mathrm{~nm}$, and by a non-oxidized Al layer substrate at its bottom. With the aim of preparation of templates with $140 \mathrm{~nm}$ diameter of pores, the first anodization was carried out in a solution of phosphoric acid $(2 \%)$ and aluminum oxalate $(0.02 \mathrm{M})$ at $195 \mathrm{~V}$ and temperature of $2-3^{\circ} \mathrm{C}$ during $6 \mathrm{~h}$. After removing the anodic layer, a second anodization process was carried out during $4 \mathrm{~h}$. Under these conditions, the top layer exhibits pores of $140 \mathrm{~nm}$ in diameter and length of $20 \mu \mathrm{m}$.

The resulting 35 and $140 \mathrm{~nm}$ AAO templates were further treated in phosphoric acid to widen the pores up to 45 and $300 \mathrm{~nm}$ of diameter, respectively.

\section{3. 'In situ' polymerization of ANI inside the AAO template}

Before carrying out the polymerization, the liquid aniline monomer was dropped little by little on top of the AAO template until formation of a uniform layer and left for infiltration into the nanocavities at room temperature. The infiltration process was carried during $45 \mathrm{~min}$ for templates of $45 \mathrm{~nm}$ of diameter and during $8 \mathrm{~h}$ for the templates of $300 \mathrm{~nm}$. Then, the excess of monomer from the aluminum was removed with sharp blades. Finally, infiltrated AAO template was quickly immersed in the $1 \mathrm{M}$ oxidant agent, ammonium peroxidedisulfate (APS) and the doping acid, hydrochloric acid ( $\mathrm{HCl} 1 \mathrm{M})$ solution and the reaction medium stirred mechanically and kept at $0{ }^{\circ} \mathrm{C}$ with the help of a refrigerating ethanol bath. The aniline monomer was polymerized with an APS concentration 1.4 times larger than ANI molar concentration, to ensure a complete reaction. Polymerization started after introduction of the template and was carried out during $2 \mathrm{~h} 30 \mathrm{~min}$.

\subsection{Infiltration of PVDF-MWCNTs films in AAO template}

In order to prepare PVDF-MWCNTs composite film, firstly, MWCNTs were separately dispersed in dimethylformamide (DMF) at a desired concentration in two steps, by magnetic stirring and ultrasonication. The temperature of the process was controlled to avoid overheating. The MWCNTs dispersion was then immediately added to the PVDF in
DMF solution while ultrasonicated continuously. Meanwhile sonication was still on, distilled water was added little by little to precipitate the solution. Afterwards, the solution was agitated to favor further precipitation. To remove the remaining DMF solvent, the polymer composite was washed with distilled water and centrifuged. The final product was dried using a lyophilization process with Telstar ${ }^{\circledR}$ Lyophilization Freeze Dry model L6-50. At the end the PVDF-MWCNTs composite presents a foamy appearance with uniform distribution of the MWCTNs and to achieve flat bulk films, all compositions were compressed using a Collins hydraulic hot press mold at a pressure of $50 \mathrm{~Pa}$ and at $210^{\circ} \mathrm{C}$ for $10 \mathrm{~min}(0.3 \mathrm{~mm}$ thick film). Infiltration of PVDFMWCNT composite was carried out in an oven under vacuum at controlled temperature by placing a PVDF-MWCNT film on the top of the AAO template. The temperature of the oven was maintained at $150^{\circ} \mathrm{C}$ during $30 \mathrm{~min}$ to evaporate any moisture from the AAO template. Then, in order to infiltrate PVDF-MWCNTs in the nanocavities, the temperature was increased and maintained at $240^{\circ} \mathrm{C}$ during $4 \mathrm{~h}$. For infiltration of PVDF, pristine PVDF pellets were placed on the templates at $200^{\circ} \mathrm{C}$ during $1 \mathrm{~h}$. The infiltration was favored by application of mechanical force.

\subsection{Characterization by scanning electron microscopy (SEM)}

The AAO templates and the infiltrated samples were morphologically characterized by SEM/TEM (Philips XL-30ESEM and FESEM Hitachi model SU8000 with TE Detector). In order to perform the analysis of free polymer nanofibers, first, the aluminum substrate was eliminated by treatment with a mixture of $\mathrm{HCl}, \mathrm{CuCl}_{2}$ and $\mathrm{H}_{2} \mathrm{O}$, and then, the alumina was dissolved in $10 \mathrm{wt} \% \mathrm{H}_{3} \mathrm{PO}_{4}$. Previously, in order to support the free nanostructures, a polymer coating was placed on the top of the template.

\subsection{Raman spectroscopy}

Bulk polymers and their polymer nanostructures inside the AAO nanocavities were characterized by Raman spectroscopy. A Renishaw In Via Raman Microscope (Renishaw plc, Wottonunder-Edge, UK) was used fitted with a grating spectrometer of 1200 lines/mm and a Peltier-cooled charge-coupled device (CCD) detector, coupled to a confocal microscope. All spectra were processed using Renishaw 
WiRE 3.3 software. For polymer nanostructures, the measurements were carried out for the filled templates, following the methodology described by Maiz et al. [30]. Briefly, the Raman scattering was excited with a $785 \mathrm{~nm}$ near-infrared diode laser of $320 \mathrm{~mW}$ maximum input power. A 100×, NA090 microscope objective lens was used to focus the laser beam with a power of $0.1 \mathrm{~W}$ on the sample, giving a laser spot diameter of $\pm 1 \mu \mathrm{m}$. With this objective the sampling depth is estimated to be around 4-5 $\mu \mathrm{m}$ (half-width of the confocal depth profile for a silicon wafer) and the lateral resolution is estimated to be about $1 \mu \mathrm{m}$ with the system operated in the confocal mode. Depth profiles were obtained by focusing the microscope stepwise, at $10 \mu \mathrm{m}$ intervals through the length of AAO templates of $45 \mathrm{~nm}$ of diameter $\times 100 \mu \mathrm{m}$ length and at $5 \mu \mathrm{m}$ intervals through AAO templates of $300 \mathrm{~nm}$ diameters $\times 20 \mu \mathrm{m}$ length.

\subsection{Broadband dielectric spectroscopy (BDS)}

A Broadband dielectric spectrometer, Novocontrol $\mathrm{GmbH}$ Concept alpha-S analyzer, was used to measure the complex dielectric function of the bulk polymers and their nanostructures inside the AAO nanocavities, in the frequency range of $10^{-2}-10^{7} \mathrm{~Hz}$ in coupling with a nitrogen-jet stream quarto cryosystem to control the temperature in the range 100 $600 \mathrm{~K}$. The samples were placed between two goldplated electrodes for AC conductivity measurements. To ensure good electrical contact with the goldplated electrodes, the PANI bulk sample was compressed $\left(10 \mathrm{t} \cdot \mathrm{mm}^{-2}, 10 \mathrm{~min}\right)$ into a thin circular film of $500 \mu \mathrm{m}$ thickness and $15 \mathrm{~mm}$ diameter.

For the polymer infiltrated AAO templates, the bottom part of aluminum layer was kept in contact with electrode, acting as a conducting electrode as well.

\section{Results and discussion}

\subsection{AAO templates}

The morphological study by SEM microscopy allows examining AAO templates. Figure 1 illustrates SEM micrographs of both kinds of synthesized alumina templates. Figures $1 \mathrm{a}$ and $1 \mathrm{~b}$ correspond to the lateral and top view of AAO templates obtained by anodization in oxalic acid. The dimensions of the nanocavities are: $43.7 \mathrm{~nm}$ of diameter, $98.3 \mathrm{~nm}$ of interpore distance and, although no shown here, around $100 \mu \mathrm{m}$ of length. Figure 1c presents the surface view of an AAO template obtained by anodization in phosphoric acid. The dimensions of the nanocavities are:

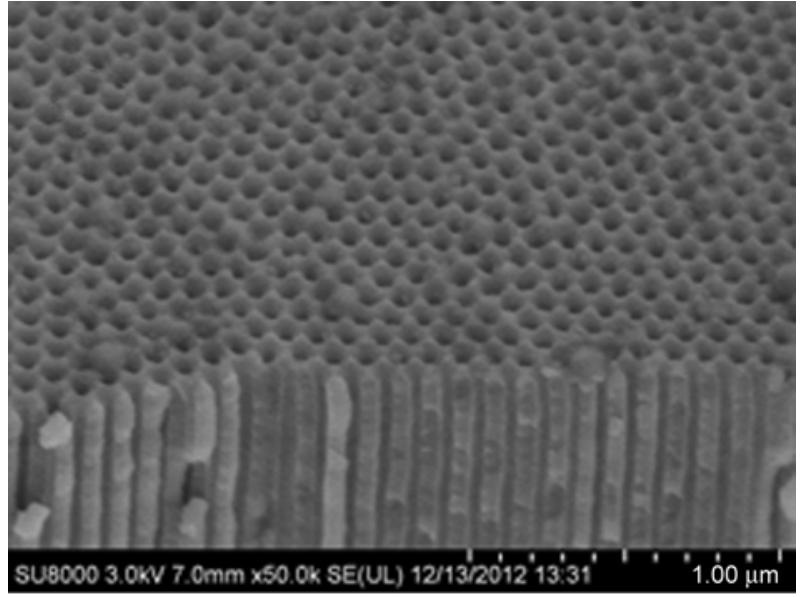

a)

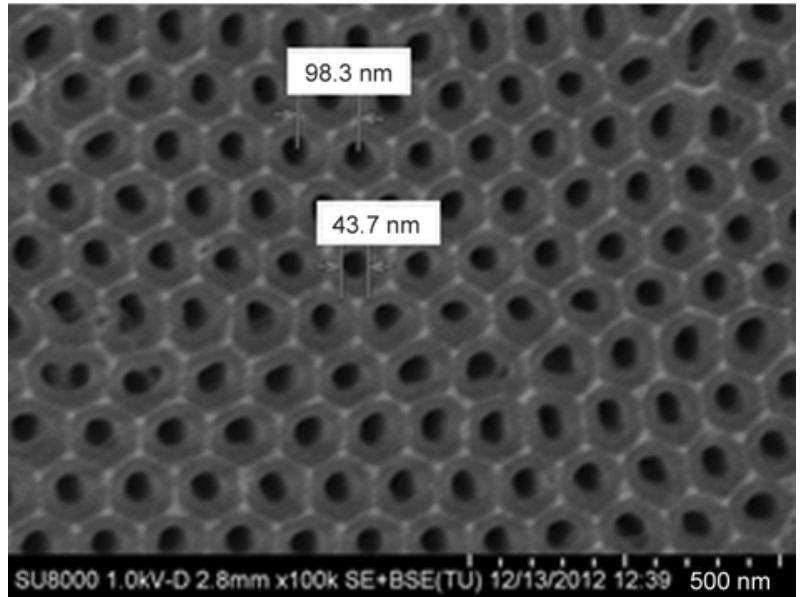

b)

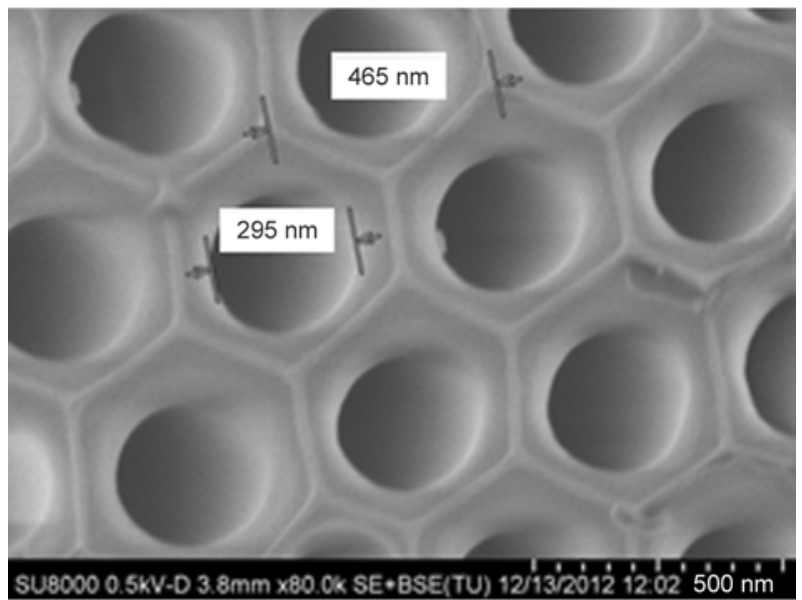

c)

Figure 1. SEM micrographs of AAO template with $45 \mathrm{~nm}$ diameter pores, lateral view (a), and top view of $45 \mathrm{~nm}$ diameter pores (b) and $300 \mathrm{~nm}$ pores (c)

$295 \mathrm{~nm}$ of diameter and $465 \mathrm{~nm}$ of interpore distance and, although no shown here, around $20 \mu \mathrm{m}$ of length. From the above images, the main characteristic of the templates can be summarized: high quality order pores, regularity in the size of the pores and invariance of the diameter throw the length of template 


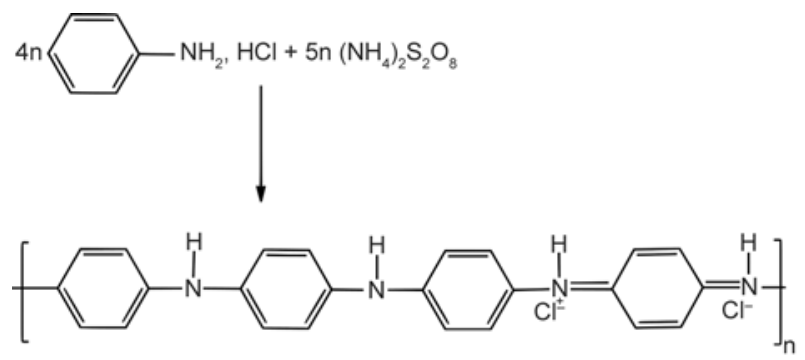

$5 \mathrm{n}_{2} \mathrm{SO}_{4}+5 \mathrm{n}\left(\mathrm{NH}_{4}\right)_{2} \mathrm{SO}_{4}+2 \mathrm{n} \mathrm{HCl}$

Figure 2. Chemical oxidative polymerization of aniline in a solution of APS/HCl to yield PANI-ES

\subsection{PANI system}

The oxidative polymerization of ANI, illustrated in Figure 2, is an exothermic process being the acid a by-product of the reaction, therefore, the course of reaction can be continuously monitored by recording the temperature and $\mathrm{pH}$ change of the solution medium. Although low temperature results in a polymer of higher molecular weight, less defects and undesirable branching, nevertheless, the polymerization time required is longer, consequently, a temperature of around $0{ }^{\circ} \mathrm{C}$ was chosen as a right compromise. This procedure was followed for polymerization in bulk and in AAO templates.

\subsection{SEM micrographs}

SEM micrographs of the one-dimensional PANI nanostructures obtained after the 'in situ' polymerization in AAO templates, once extracted from the templates, are collected in Figure 3. Figure 3a corresponds to PANI nanostructures obtained in AAO templates of $45 \mathrm{~nm}$ of diameter $\times 100 \mu \mathrm{m}$ of length and Figure $3 b$ and $3 c$ to those obtained in AAO of $300 \mathrm{~nm} \times 20 \mu \mathrm{m}$. In both cases and up to several tens of microns, it is observed that diameter and length size of nanofibers pattern the dimensions and symmetry of AAO. Nevertheless, some differences between them are found. For PANI nanostructures obtained in AAO nanocavities of dimensions $300 \mathrm{~nm}$ $\times 20 \mu \mathrm{m}$ (Figure $3 \mathrm{~b}$ and $3 \mathrm{c}$ ) it can be clearly observed free-standing arrays of nanopillars. The determining factor for achieving such free-standing nanostructures is the low aspect ratio of nanostructure (about 60 ), although other factors such as rigidity, surface chemistry and electrostatic interactions could be also taken into consideration. For PANI nanostructures obtained in AAO nanocavities of dimension $45 \mathrm{~nm} \times 100 \mu \mathrm{m}$ (Figure 3a) it is clearly noticed that PANI nanofibers tend to collapse. The collapse is certainly due to their high aspect ratio (order of magni-

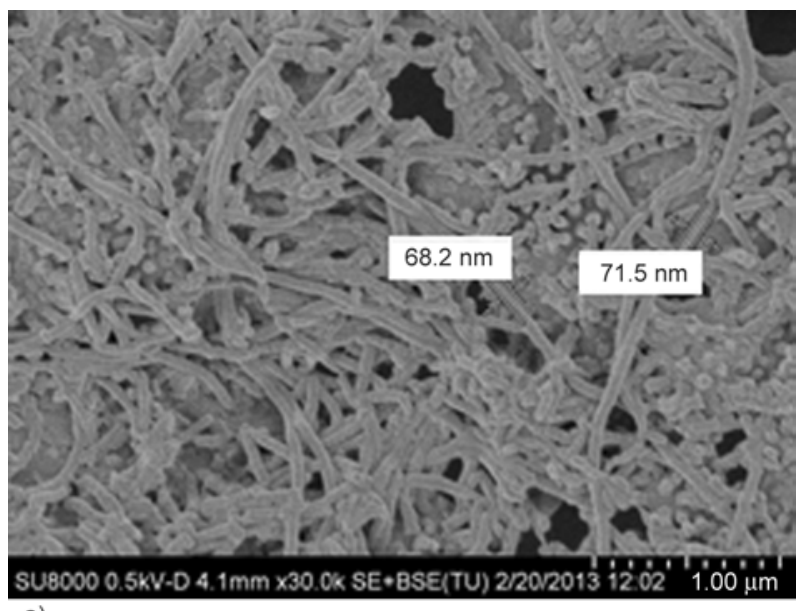

a)

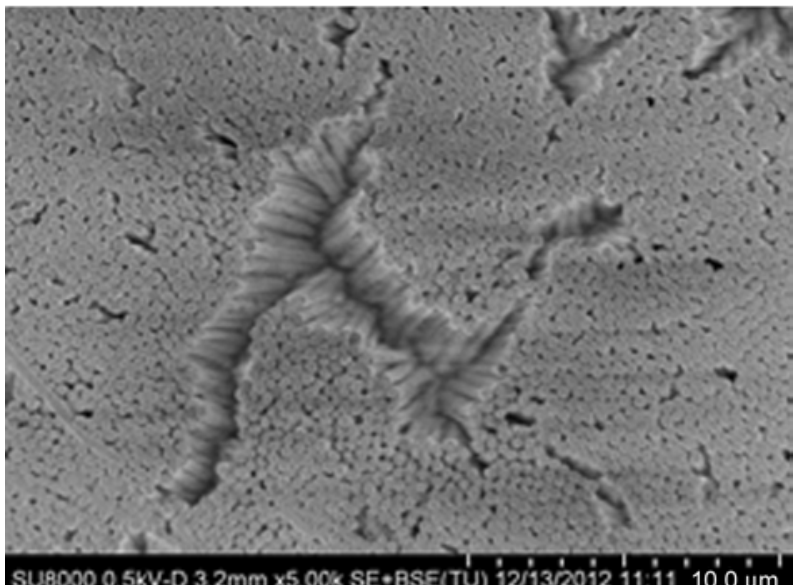

b)

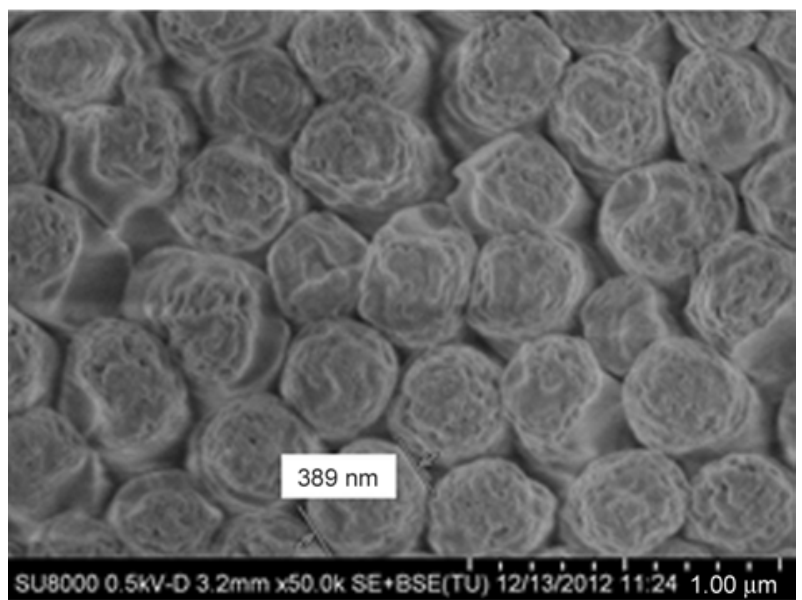

c)

Figure 3. Different magnifications of PANI into $45 \mathrm{~nm}$ AAO pores (a), and $300 \mathrm{~nm} \mathrm{AAO} \mathrm{pore} \mathrm{(b} \mathrm{and} \mathrm{c)}$

tude 2300). Moreover, it results in an increase in the diameter of nanofibers (to around $70 \mathrm{~nm}$ ) in comparison to that of template $(45 \mathrm{~nm})$. This fact can be explained by the agglomeration of two separated fibers as a consequence of the surface tension and the interaction between individual nanopillars.

$\mathrm{Be}$ as it may, PANI nanostructures obtained by polymerization pattern the dimensions of AAO templates, 
so their final dimensions can be adjusted as a function of the size of AAO nanocavities.

\subsection{Raman spectroscopy}

Raman spectroscopy was used to determine if PANI was polymerized correctly in the pores of the template and to check if the conductive (emeraldine salt, ES) form was obtained. First, the Raman spectrum of PANI bulk was analysed to serve as a reference, Figure 4a). The analysis of main regions of PANI-ES and their assignments are supported by data from other works [31-34]. The zone of most interest is the region $1000-1700 \mathrm{~cm}^{-1}$. There are two dominating
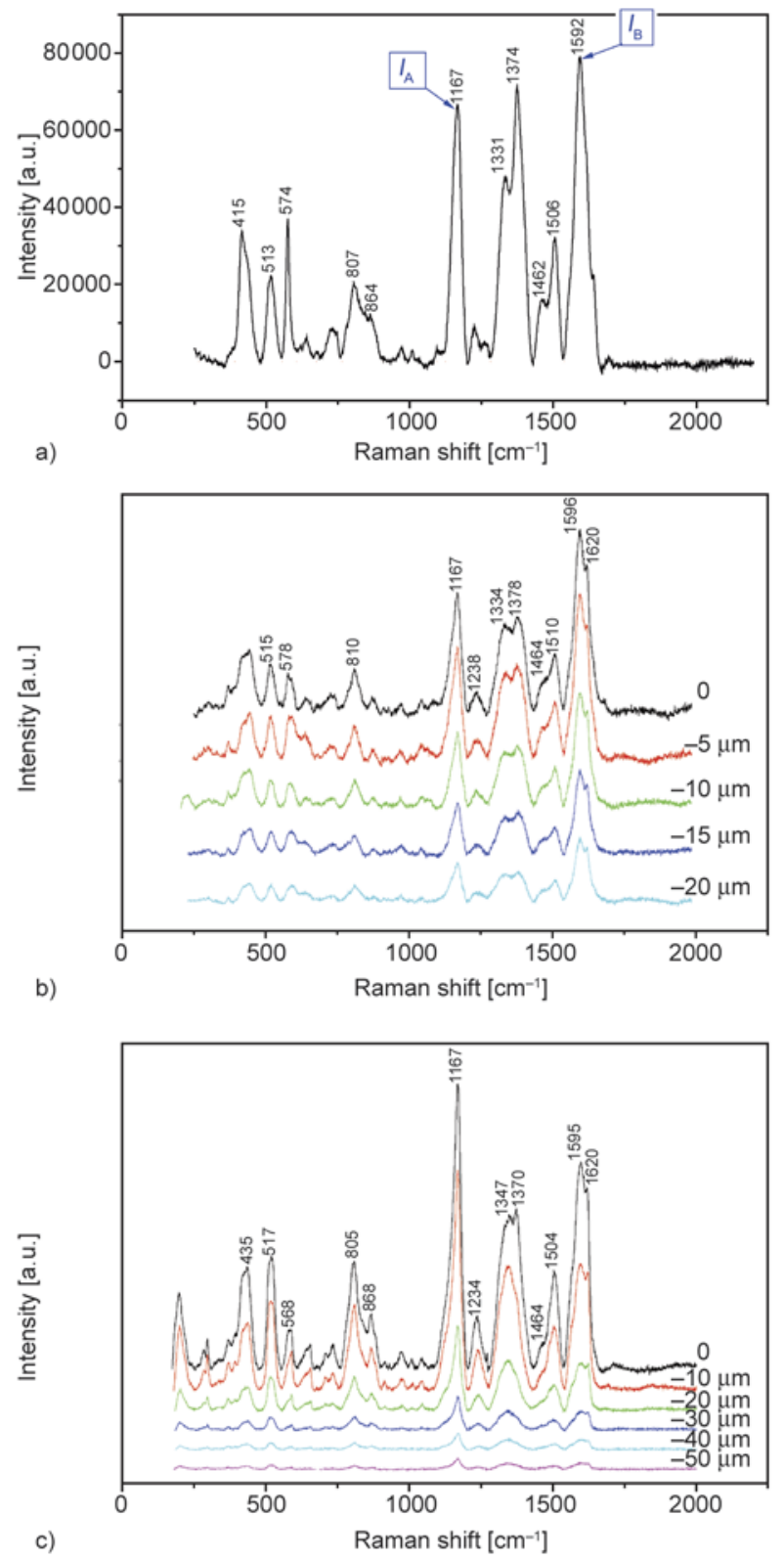

Figure 4. Raman spectrum of PANI bulk (a), PANI polymerized in AAO templates of $300 \mathrm{~nm}$ (b) and idem in $45 \mathrm{~nm}$ (c) bands in the spectra, one corresponds to $v(\mathrm{C}=\mathrm{C})_{\mathrm{Q}}$ vibration of quinoid ring localized at $1595-1597 \mathrm{~cm}^{-1}$ and the other corresponds to the $\delta(\mathrm{C}-\mathrm{H})_{\mathrm{Q}}$ bending in-plane vibration of quinoid ring localized at $1167-$ $1169 \mathrm{~cm}^{-1}$.

The confocal Raman spectra of PANI polymerized in AAO nanocavities of 300 and $45 \mathrm{~nm}$, were recorded every 5 and $10 \mu \mathrm{m}$ and plotted in Figure $4 \mathrm{~b}$ and $4 \mathrm{c}$, respectively.

The multiple scans every 5 and $10 \mu \mathrm{m}$ in depth for PANI obtained into AAO of 300 and $45 \mathrm{~nm}$, respectively, prove that the polymerization has been successful down to $20 \mu \mathrm{m}$ (the length of AAO template) or $50 \mu \mathrm{m}$ (the limit of confocal detection), respectively. The main difference between the spectra of these samples lies in the relative intensity of the main bands corresponding to $(\mathrm{C}-\mathrm{H})_{\mathrm{Q}}\left(1167 \mathrm{~cm}^{-1}\right)$ and $(\mathrm{C}=\mathrm{C})_{\mathrm{Q}}\left(1592 \mathrm{~cm}^{-1}\right)$. For PANI polymerized in AAO templates of $300 \mathrm{~nm}$, the relationship between them is $<1$ and close to PANI bulk, but, on the contrary, for PANI polymerized in AAO of $45 \mathrm{~nm}$ the intensity of band $(\mathrm{C}-\mathrm{H})_{\mathrm{Q}}$ is much higher than the intensity of $(\mathrm{C}=\mathrm{C})_{\mathrm{Q}}$. This difference can be explained in terms of the orientation of the polymer chains and confinement effects. Confinement effects would bring about a change in the intensity of the orientation of the chains. Indeed, orientation affects the intensity of the Raman scattered-light for a given polarization of the incident light [35]. Owing to the smaller pore diameter, uniaxial symmetry is more pronounced for polyaniline obtained in AAO of 45 $\mathrm{nm}$ than of $300 \mathrm{~nm}$, so the polymer chains tend to have a preferred orientation (parallel to the AAO walls) [36] thus increasing the intensity of the band at $1167 \mathrm{~cm}^{-1}$, as observed.

To summarize, the spectra of PANI obtained in pores of $300 \mathrm{~nm}$ diameter present the same tendency than that of PANI bulk while spectra of PANI obtained in pores of $45 \mathrm{~nm}$ illustrate a confinement effect which is not seen in $300 \mathrm{~nm}$. Moreover, from the quantitative analysis of spectra it can be inferred that ANI polymerization takes place quantitatively along the length of nanocavities, in agreement with SEM results.

\subsection{PVDF-MWCNTs system}

The preparation of PVDF-MWCNTs composites and the chemical characterization by Raman spectroscopy is described in experimental part. The morphological characterization by SEM spectroscopy of PVDF- 
MWCNT (3\%) nanofibers, once extracted from the templates, is shown in Figure 5. Figure 5a corresponds to PVDF-MWCNT nanostructures obtained in AAO templates of $45 \mathrm{~nm}$ and Figure $5 \mathrm{~b}$ and $5 \mathrm{c}$ to nanostructures from templates of $300 \mathrm{~nm}$. As in PANI case, Figure $5 \mathrm{~b}$ and $5 \mathrm{c}$ shows a compact array of numerous PVDF-MWCNTs nanopillars with a perfectly defined geometry and big diameter. Once more, for nanocavities of $300 \mathrm{~nm}$ diameter and $20 \mu \mathrm{m}$ length, the determining factor for achieving such freestanding nanostructure is due to the low aspect ratio of nanorod (about 60) and also the rigidity of the polymer. On the contrary and similar to PANI case, for AAO nanocavities of high aspect ratio $(45 \mathrm{~nm}$ diameter and $100 \mu \mathrm{m}$ length), Figure 5a shows an agglomeration of non-ordered PVDF-MWCNTs nanofibers. Here again, although the composite polymer is rigid, the high aspect ratio of nanofiber (around 2300) makes the nanofiber cannot stand up and collapse. In this case, is not observed a tendency of nanopillars to agglomerate like in the case of PANI.

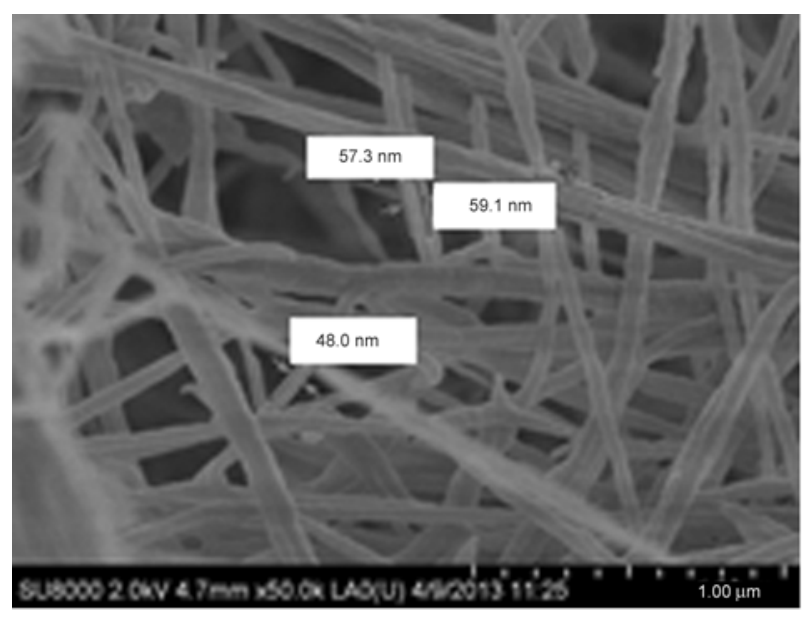

a)

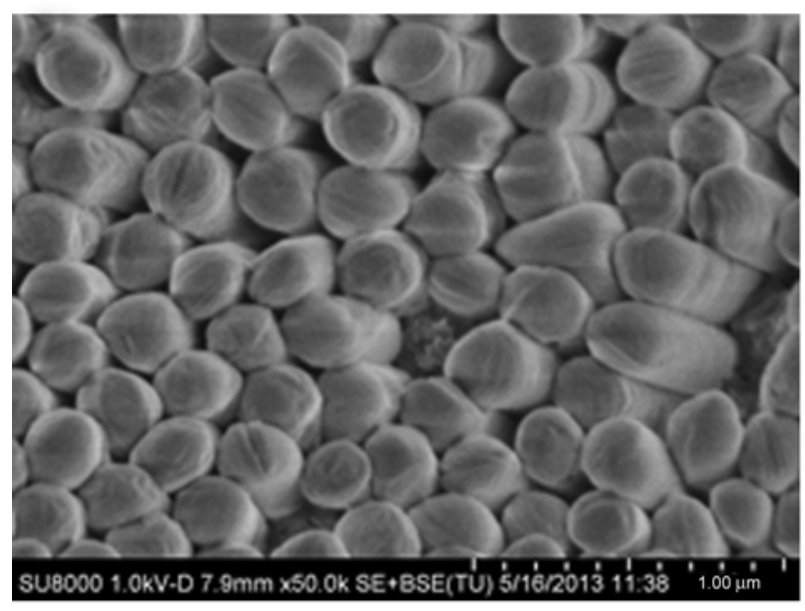

c)
Moreover, on Figure 5d we can easily observe nanofibers of PVDF with fragmented carbon nanotubes. It is worth mentioning that some of fibers are without MWCNTs due to the small diameter of nanofiber $(45 \mathrm{~nm})$ and quite variety in diameter of carbon nanotubes (20-60 nm).

Therefore, PVDF-MWCNTs and PANI nanostructures with modulated morphologies, i.e. long nanofibers, short nanopillars, etc., can be easily achieved from anodized aluminum oxide (AAO) templates assisted methods. Moreover, the fact that PVDFMWCNTs and PANI can be adjusted to nanoscopic dimensions, allow the study of size-conducting properties dependency in the nanoscale.

\subsection{Broadband dielectric spectroscopy}

BDS was the tool used to analyze the conductivity of the PANI and PVDF-MWCNTs samples in bulk and confined in AAO templates. The parameter of interest for this study is the DC conductivity $\sigma^{\prime}(\omega)$ which can be extrapolated from AC conductivity at

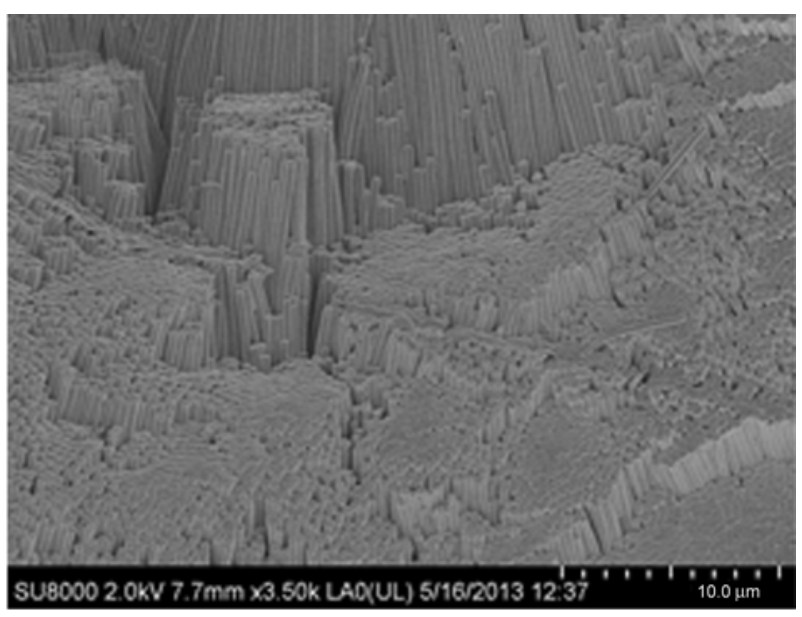

b)

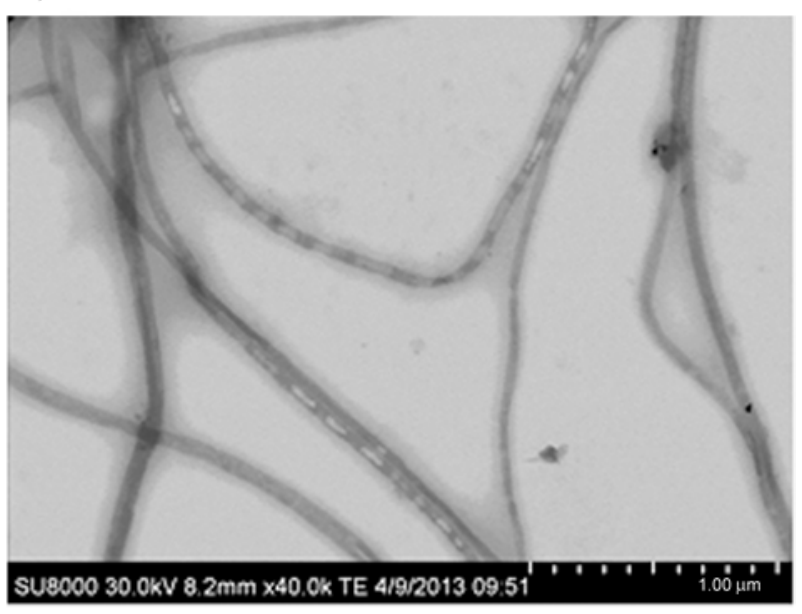

d)

Figure 5. PVDF-MWCNTs 3\% inside $45 \mathrm{~nm}$ (a) and $300 \mathrm{~nm}$ (b and c) AAO pores. TEM micrographs of PVDF-MWCNTs nanofibers with diameter of $45 \mathrm{~nm}(\mathrm{~d})$. 
low frequencies $\left(\sigma_{\mathrm{DC}} \approx \sigma^{\prime}(\omega \rightarrow 0)\right)$. Consequently, only a small part of the dielectric data is of practical use.

\subsubsection{PANI system}

Figure 6a plots the conductivity of bulk PANI as a function of the frequency at a few key temperatures. The results clearly prove that doping has been effective because, at room temperature, conductivities achieved values of the order of $10^{-2} \mathrm{~S} \cdot \mathrm{cm}^{-1}$. It also indicates that strongly doped samples behave like a metal and the real component of conductivity is frequency-independent in the whole range. Moreover, no contributions from interfacial polarization can be seen. The thermal behavior of conductivity is shown on Figure $6 \mathrm{~b}$ and corresponds to the typical of a semiconductor. As observed in the figure, conductivity increases exponentially with temperature as hopping of carriers and diffusion of ions is favored. The arrows indicate that at first, the temperature was progressively increased during the measurement to a maximum of $120^{\circ} \mathrm{C}$ and then decreased. Differences between two equivalent points can be explained by influence of atmosphere. Water is easily absorbed from the atmosphere and always presents in PANI samples so, in consequence, influences on ion transport and enhances conductivity. From this fact, it is reasonable to conclude that the conduction mechanism in doped PANI system is both electronic and ionic in nature. This procedure of temperature increase was applied for all the following measurements, therefore, it can assured that the conduction values are not influenced by the amount of absorbed water. To support this analysis, similar results were found in the literature in the study of the influence of water molecules over EB [37]. In that work, a positive temperature coefficient, $\partial \sigma / \partial T>0$, also for high doping levels, was evidenced.

The variation of temperature in the PANI electrical transport data, in the range (223-393 K), is analyzed with the Mott VRH equation (Equation (1)) [38]:

$\sigma(T)=\sigma_{0} \exp \left[-\left(\frac{T_{0}}{T}\right)^{\gamma}\right]$

where $\sigma_{0}$, a prefactor, $T_{0}$, a characteristic temperature including details of the system (density of states at Fermi level, number of nearest neighbor hopping site) and $\gamma=1 /(d+1)$, where $d$ is the effective dimensionality of the system. Hence $d=3$ for a $3 \mathrm{D}$ system, $d=2$ in $2 \mathrm{D}$ and $d=1$ in 1D. When con-
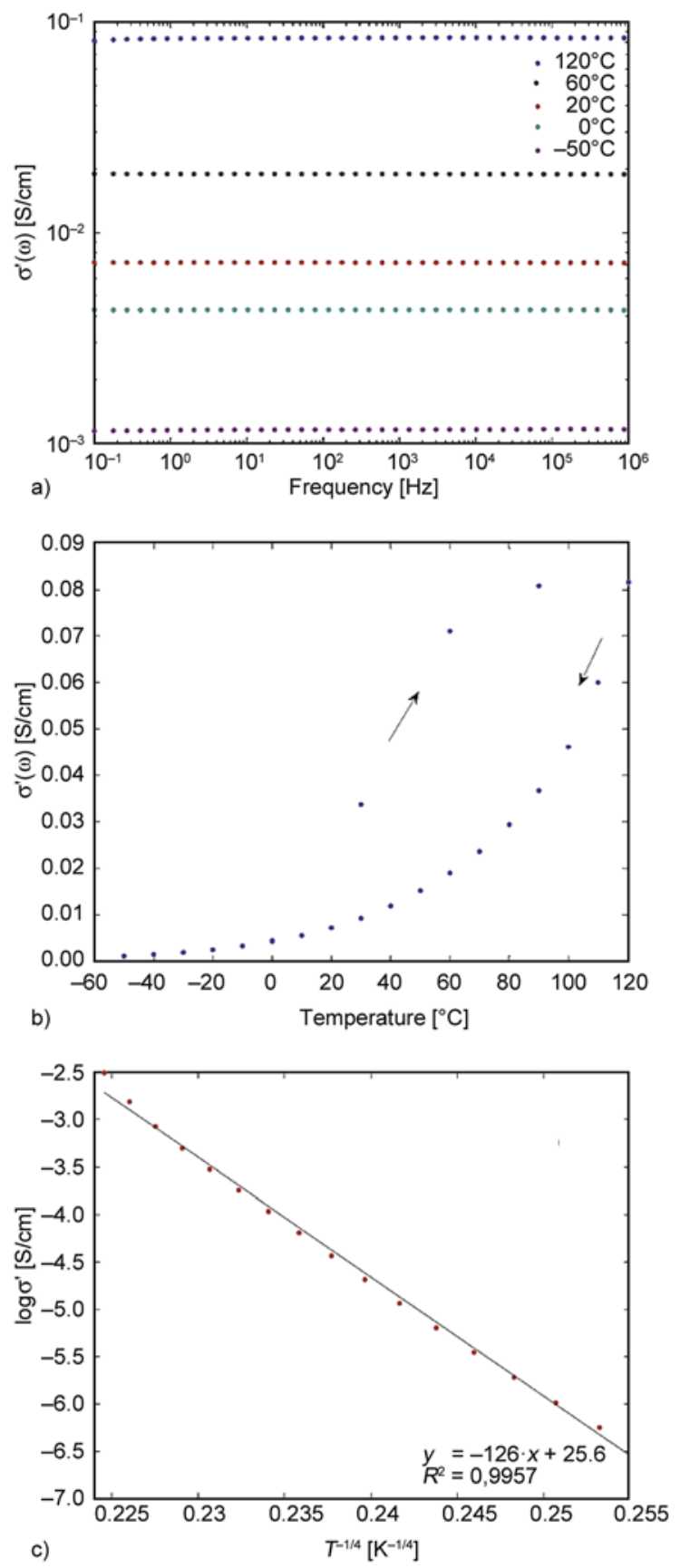

Figure 6. Conductivity of PANI bulk film versus frequency for different temperatures (a) and versus temperature at 0.1 Hertz (b). $\log \sigma$ vs $T^{-0.25}$ with linear fitting for the PANI bulk film at 0.1 Hertz (c).

fronted to a one-dimensional nanostructure, the dimensionality is affected by its diameter. Below a certain diameter, $1 \mathrm{D}$-conduction occurs owing to the large proportion of ordered material. In our case (bulk PANI), the Mott VRH equation (Equation (1)) was best fitted with $\gamma=0.25$ for a $3 \mathrm{D}$ system as can be seen in Figure 6c. This result clearly demonstrates a thermally-activated process. Moreover, Zhang et al. [39] reported that for slightly doped 
PANI salt films with different dopants ( $\mathrm{HCl}, \mathrm{CSA}$ and DBSA), the increase of conductivity follows the increase of doping level, reaching a threshold for $\sigma>10^{-2}$ at $10^{-1} \mathrm{M}$ acid concentration (transition insulator-metal). These results also show conductivity independence of the frequency as in our study. Nevertheless, these authors [39] presented a negative real permittivity $q^{\prime}$ over the whole range while we only observed it at low frequencies.

PANI nanofibers inside the AAO templates were also studied by BDS. Figures $7 \mathrm{a}$ and $7 \mathrm{~b}$ plot the conductivity of PANI polymerized in AAO nanocavities of $45 \mathrm{~nm}$ as a function of frequency and temperature, respectively and (Figures $7 \mathrm{c}$ and $7 \mathrm{~d}$ ), the same plots for PANI polymerized in nanocavities of $300 \mathrm{~nm}$. In both Figures, the red continuous line corresponds to an empty AAO template, being a reference line to evaluate the conduction response. This line clearly increases with frequency as can be expected from alumina, an insulator. The blue points

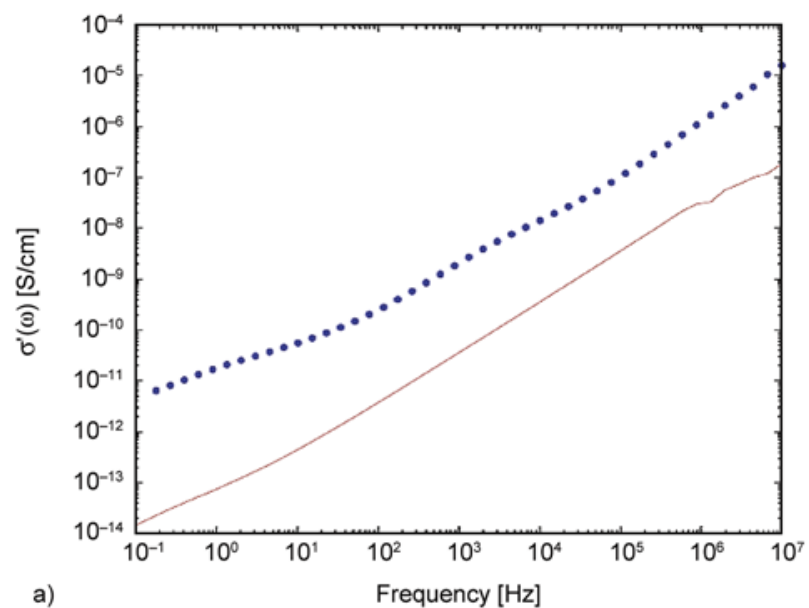

a)

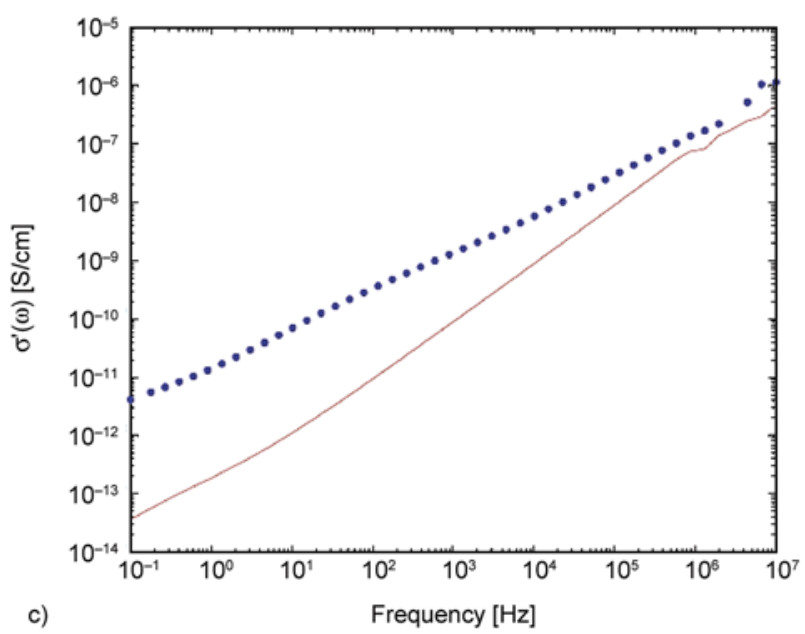

are the experimental data and are situated above the reference line. From the comparison of both lines, an increment of conductivity is observed although probably lower to the real one, since the barrier layer present at the bottom of the AAO pores could act as a thin insulating that would impede electrons to fully go through. Both type of PANI filled templates exhibits higher conductivity by 2 orders of magnitude in comparison with empty template. Moreover, there is very little difference between the conductivity value for PANI in AAO pores of 45 and for PANI in $300 \mathrm{~nm}$, being $8 \cdot 10^{-12}$ and $6 \cdot 10^{-12} \mathrm{~S} \cdot \mathrm{cm}^{-1}$, respectively. In agreement with Martin and coworkers [18, 19], who observed 3 times higher conductivity for nanostructured PANI, one would expect that PANI nanofibers in AAO templates of $45 \mathrm{~nm}$ were more conductive than PANI nanofibers in AAO templates of $300 \mathrm{~nm}$. Nevertheless, in agreement to our results, Wu et al. [22] found that for PANI nanowires and nanorods the electrical conductivity values were
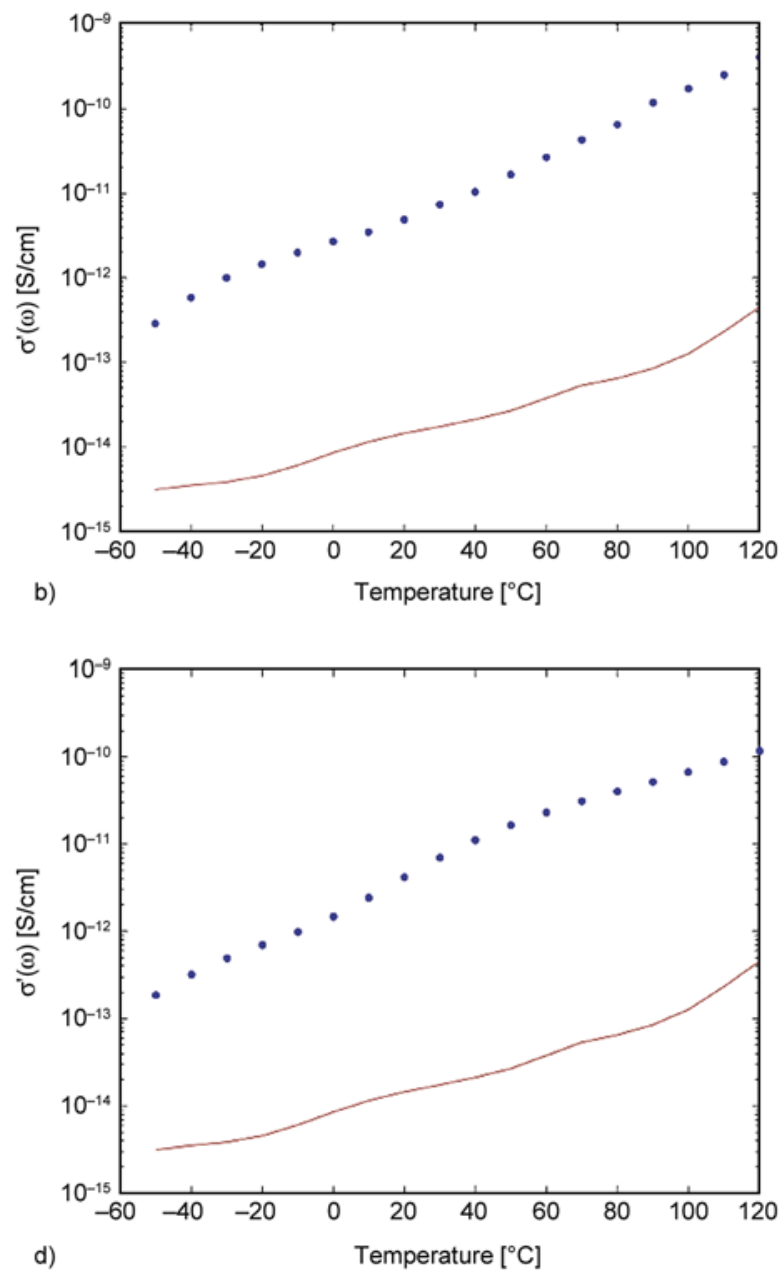

Figure 7. Conductivity of AAO template filled with PANI as a function of frequency, at room temperature for template of $45 \mathrm{~nm}$ (a) and for $300 \mathrm{~nm}$ (c) and as a function of temperature at 0.1 Hertz for template of $45 \mathrm{~nm}$ (b) and $300 \mathrm{~nm}$ (d). Empty AAO template is used as a reference (red line). 
similar, 1.25 and $2.06 \mathrm{~S} \cdot \mathrm{cm}^{-1}$, respectively, so the difference found in both types of structures is similar to that found by us. In our case, the rather higher conductivity value obtained for PANI in nanopores of $45 \mathrm{~nm}$ could be due to the thinner barrier layer of AAO template of $45 \mathrm{~nm}$ compared to that of $300 \mathrm{~nm}$.

\subsubsection{PVDF-MWCNTs system}

Figure 8 displays the conductivity variation of bulk PVDF-MWCNT films with different weight percentages of MWCNT, $0 ; 1 ; 3$ and $5 \%$. It is obvious that a small addition of CNTs increases the conduction by more than 10 orders of magnitude compared to pure PVDF. At room temperature, bulk PVDF has a conductivity of $10^{-14} \mathrm{~S} \cdot \mathrm{cm}^{-1}$ while for PVDFMWCNT $(1 \%)$ is $3 \cdot 10^{-3} \mathrm{~S} \cdot \mathrm{cm}^{-1}$, similar to that obtained for polyaniline. For higher MWCNT content, higher conductivity, but the increase is quite small compared to the change in concentration. Tripling the quantity of MWCNT (from 1 to $3 \mathrm{wt} \%$ ), the conductivity increases only 2 orders of magnitude, thus indicating that a percolation threshold has already been reached at $1 \mathrm{wt} \%$. Nevertheless, studies do not agree on the percolation threshold [4044] since there is no unique value, as it depends on the process conditions, the possible preferred orientation of the CNTs in the matrix, the CNTs type and their characteristics (i.e. AR, surface modification [45-48]). In the case of a $5 \mathrm{wt} \%$ of MWCNT, the conductivity still increases although flexibility and processability of the polymer is lost as reported by Lonjon et al. [49] in the case of P(VDF-TrFE) and nickel nanowires. For these two opposing reasons, only PVDF-MWCNT (3\%) in bulk and infiltrated in AAO templates will be deeply studied by BDS.

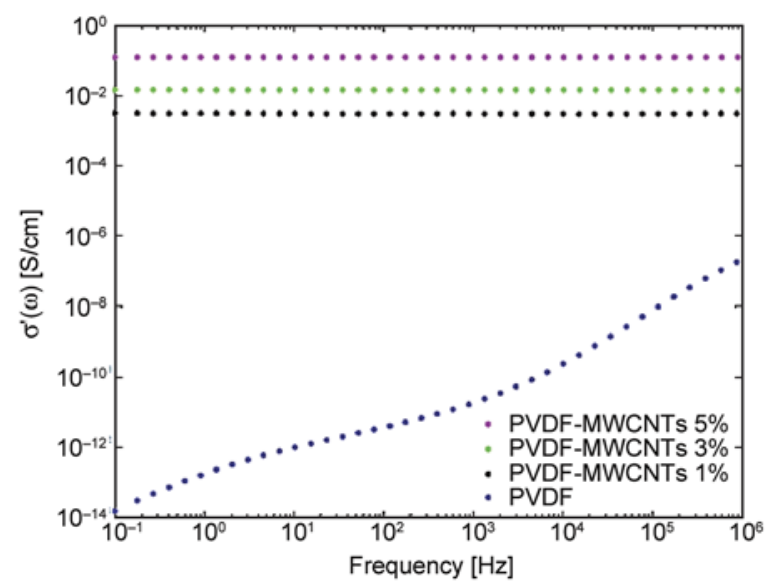

Figure 8. AC conductivity of PVDF-MWCNTs films at different weight percentages of MWCNT at room temperature
Figure 9 presents the isochrones of PVDF-MWCNT (3\%) films at the lowest measured frequency $(0.1 \mathrm{~Hz})$. The conductivity evolution of the nanocomposite in function of temperature is composed of two different regimes. Below $100^{\circ} \mathrm{C}$ the evolution is characteristic of a semiconductor with the conductivity increasing with temperature $(\partial \sigma / \partial T>0)$. Over $100^{\circ} \mathrm{C}$, there is a switch and conductivity behaves like a metal, decreasing with increasing temperature $(\partial \sigma / \partial T<0)$. In our case $\left(T<T_{\mathrm{m}}\right)$, we are faced with a static and immobilized conductive network of MWCNTs in the polymer matrix since we are in the percolation region and at low temperatures. To explain the results we presume that, at first, the tunneling of the electrons between adjacent carbon nanotubes is favored by the increase of temperature. The electrons gain thermal energy which helps them to overcome the potential barrier more easily, as supported by the FIT model. However, at a temperature limit of $100^{\circ} \mathrm{C}$, for this PVDF-MWCNT (3\%) composite the thermal expansion of the polymer matrix compensates the thermally-induced tunneling of MWCNT. This can be explained by the fact that increasing the average distance between nanotubes, the amount of insulating polymer becomes significant and makes a wider barrier that electrons struggle overpass, hence a reduction in the conductivity with further increase of temperature would be observed. Moreover, in semicrystalline polymers, the volume expansion of the polymer matrix which separates the fillers is also attributed to the transformation of the crystalline phase to the amorphous phase. These results agree with others reported in the literature [50]. For instance Tao et al. [51] also reported a decrease in conductivity with temperature for a composite of MWCNTs and polyethylene at higher temperatures $\left(T>T_{\mathrm{m}}\right)$ and was related to the phenomena of crystallization of polyethylene which would disconnect the conductive paths [51]. Other authors [40,52] also observed a continuous increase of conductivity with temperature below $T_{\mathrm{m}}$ (positive temperature coefficient effect of the resistivity). Moreover, an effect similar was reported by Li et al. [50] below a transition temperature of $80^{\circ} \mathrm{C}$. This effect was explained by the fact that at higher temperatures $\left(T>T_{\mathrm{m}}\right)$, Brownian motion of the carbon nanotubes make them mobile within the melting matrix and they tend to coagulate which results in an decrease of the electrical conductivity of the composite with temperature. 


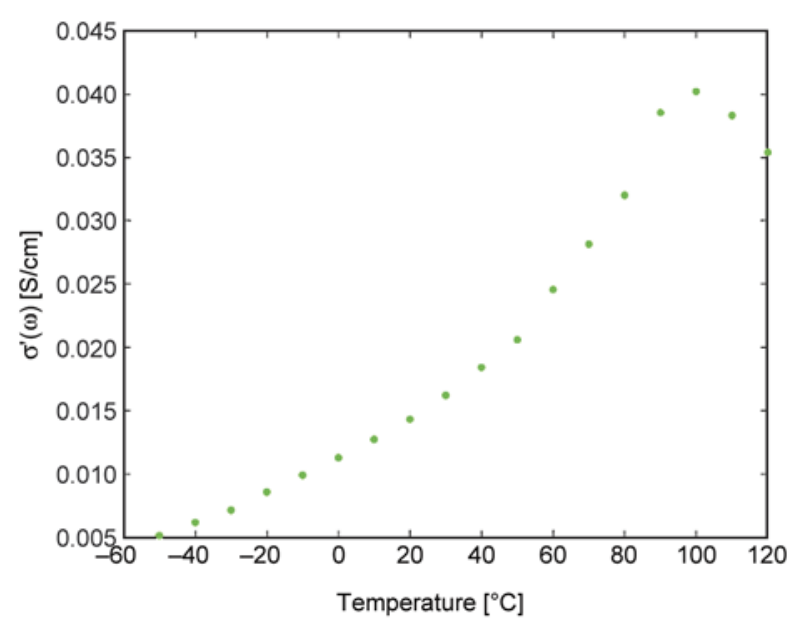

Figure 9. Conductivity versus temperature of PVDF-MWCNTs films at $0.1 \mathrm{~Hz}$ for a $3 \%$ CNT content

Figure 10 presents the conductivity of two AAO templates filled with the nanocomposite of PVDFMWCNT 3\%, at room temperature. Figure 10a accounts for the template of $45 \mathrm{~nm}$ of diameter; the template filled with PVDF and with PVDF-MWCNT $3 \%$. It is observed that infiltrated conducting nanocomposite in AAO exhibits higher conductivity than the PVDF infiltrated alone and evidences the rather surprising fact that carbon nanotubes with an average diameter of $40 \mathrm{~nm}$ could penetrate the small pores. Figure $10 \mathrm{~b}$ accounts for the templates of $300 \mathrm{~nm}$ of diameter and as observed do not show a significant improvement of conductivity. The above mentioned effect of the insulating barrier layer could explain the low response.

Comparing the conductivity values of polymer nanostructures of PVDF-MWCNT 3\% and of PANI, in AAO templates of 45 and $300 \mathrm{~nm}$ diameters, the difference is the same as that found between the two

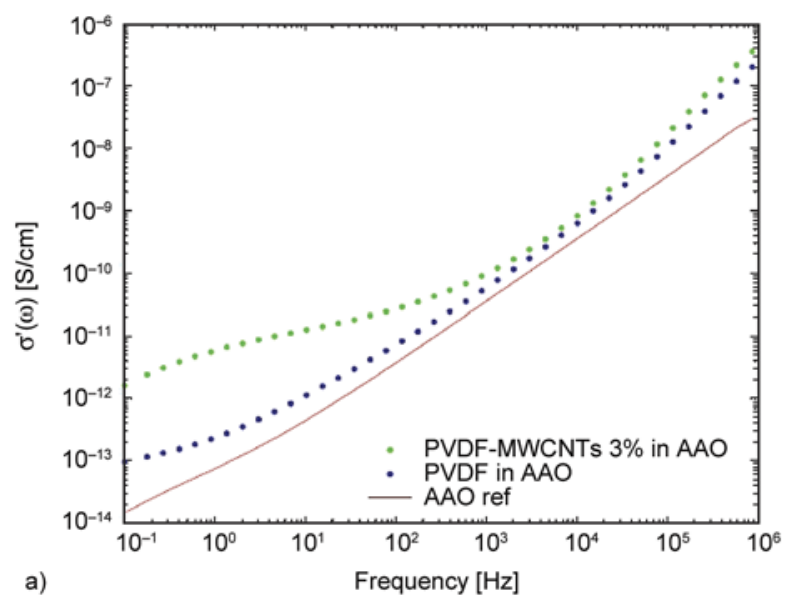

bulk polymers. So, the same conductivity is obtained for a nanostructured ECP (PVDF with a 3\% of MWCNT) than for a nanostructured ICP (PANI). Therefore, the above results evidence that BDS is an easy and non-destructive method to determine electrical conductivity of PANI and PVDF-MWCNT polymer nanostructures confined in AAO templates. Moreover, this example could be extended to other nanoscopic conducting polymer systems

\section{Conclusions}

The core of this work was the synthesis of onedimensional polymer nanostructures of PANI and PVDF-MWCNT within AAO templates to combine performance and conductivity down to nanoscale and we present an accurate way of increasing and modeling the conductivity of two different kinds' polymeric materials by nanostructuration that opens huge possibilities in the technologies of the future. Conductivity of bulk materials was increased substantially by several orders of magnitude compared to a classic insulating polymer either by doping the intrinsic conducting polymer, polyaniline, or by incorporating conductive nanofillers into poly (vinylidene fluoride). It was demonstrated that by using AAO template it was possible to prepare different kinds of high quality conductive nanomaterials with variable diameters of nanoarray $(45-300 \mathrm{~nm})$. The nanostructures were obtained successfully inside the templates either by a simple chemical method of polymerization of aniline or by melt infiltration of the PVDF-MWCNT nanocomposite. Polymerization and infiltration parameters were found to be critical and needed to be well-controlled to achieve the desired morphology. Raman spectroscopy used in

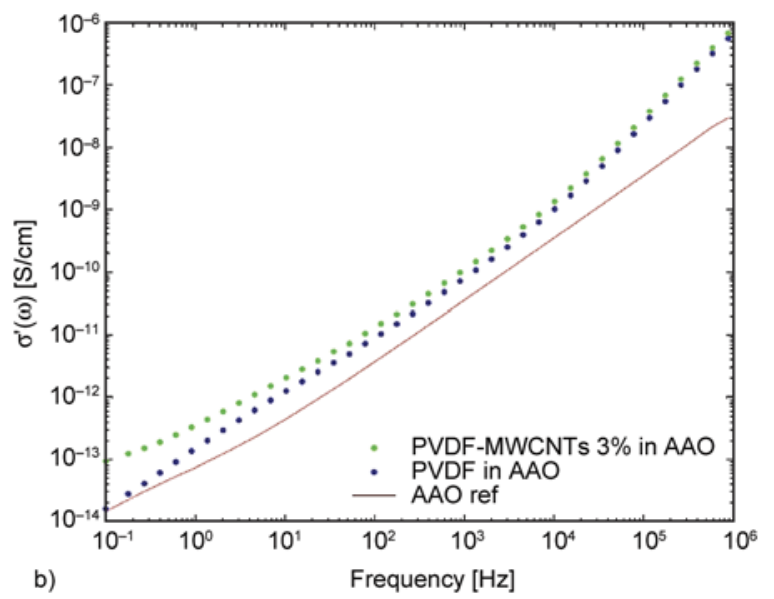

Figure 10. Conductivity of filled AAO template with PVDF-MWCNTs 3\% at room temperature for $45 \mathrm{~nm}$ pore diameter (a) and for $300 \mathrm{~nm}$ (b) 
confocal mode detected that the emeraldine salt of PANI was obtained correctly along the length of the nanopores of the AAO template.

For the first time, to our knowledge, dielectric spectroscopy was used to determine in a non-destructive way the conductivity of nanostructures present within the AAO templates. Thus, control over the concentration of dopant for PANI as well as of the filler concentration for PVDF-MWCNT makes possible the tuning of the level of conduction.

In summary, infiltration of a conducting polymer in a lab-made anodized aluminum oxide template is an effective method to produce well-defined one-dimensional nanostructures with regular spatial arrangement and remarkably low polydispersity. Besides, the possibility of precise control of aspect ratio allows adjusting to the application needs. Once free of the template, this kind of compact conducting nanoarray is of great interest for sensing or field emission display as they could be addressed individually. This new approach for rapid evaluation of conductivity of nanomaterials, however, does not give objective value due to the insulating properties of templates.

\section{Acknowledgements}

Financial support from the Spanish 'Ministerio de Economia y Competitividad' under projects MAT2011-24797 and MAT2014-53437-C2-1 is acknowledged. The authors thank D. Gómez for SEM experiments and I. Muñoz for Raman spectra. Authors thank Miguel Ángel López Manchado por providing MWCNTs.

\section{References}

[1] Long Y-Z., Chen Z., Gu C., Wan M., Duvail J-L., Liu Z., Ringer S. P.: A review on electronic transport properties of individual conducting polymer nanotubes and nanowires. in 'Nanowires science and technology' (ed.: Lupu N.) InTech, Rijeka, 223-242 (2010).

DOI: $10.5772 / 39488$

[2] Wang Y. Y., Jing X. L.: Intrinsically conducting polymers for electromagnetic interference shielding. Polymers for Advanced Technologies, 16, 344-351 (2005). DOI: $10.1002 /$ pat.589

[3] Koval'chuk A. A., Shchegolikhin A. N., Shevchenko V. G., Nedorezova P. M., Klyamkina A. N., Aladyshev A. M.: Synthesis and properties of polypropylene/multiwall carbon nanotube composites. Macromolecules, 41, 3149-3156 (2008).

DOI: $10.1021 / \mathrm{ma} 800297 \mathrm{e}$

[4] Saini P., Choudhary V.: Electrostatic charge dissipation and electromaghetic interference shielding response of polyaniline based conducting fabrics. Indian Journal of Pure and Applied Physics, 51, 112-117 (2013).
[5] Vecino M., González I., Muñoz M. E., Santamaría A., Ochoteco E., Pomposo J. A.: Synthesis of polyaniline and application in the design of formulations of conductive paints. Polymers for Advanced Technologies, 15, 560-563 (2004).

DOI: $10.1002 /$ pat.502

[6] Griesser T., Radl S. V., Koepplmayr T., Wolfberger A., Edler M., Pavitschitz A., Kratzer M., Teichert C., Rath T., Trimmel G., Schwabegger G., Simbrunner C., Sitter H., Kern W.: UV-induced modulation of the conductivity of polyaniline: Towards a photo-patternable charge injection layer for structured organic light emitting diodes. Journal of Materials Chemistry, 22, 2922-2928 (2012).

DOI: $10.1039 / \mathrm{c} 1 \mathrm{jm} 14100 \mathrm{~g}$

[7] Peng H., Zhang L., Soeller C., Travas-Sejdic J.: Conducting polymers for electrochemical DNA sensing. Biomaterials, 30, 2132-2148 (2009).

DOI: 10.1016/j.biomaterials.2008.12.065

[8] Pramanik S., Das G., Karak N.: Facile preparation of polyaniline nanofibers modified bentonite nanohybrid for gas sensor application. RSC Advances, 3, 4574- 4581 (2013).

DOI: $10.1039 / \mathrm{c} 3 \mathrm{ra} 22557 \mathrm{~g}$

[9] Li G., Li Y., Li Y., Peng H., Chen K.: Polyaniline nanorings and flat hollow capsules synthesized by in situ sacrificial oxidative templates. Macromolecules, 44, 93199323 (2011).

DOI: $10.1021 / \mathrm{ma} 2014854$

[10] Wang W., Schiff E. A.: Polyaniline on crystalline silicon heterojunction solar cells. Applied Physics Letters, 91, 133504/1-133504/3 (2007).

DOI: $10.1063 / 1.2789785$

[11] Zhang J., Hreid T., Li X., Guo W., Wang L., Shi X., Su H., Yuan Z.: Nanostructured polyaniline counter electrode for dye-sensitised solar cells: Fabrication and investigation of its electrochemical formation mechanism. Electrochimica Acta, 55, 3664-3668 (2010). DOI: $10.1016 /$ j.electacta.2010.01.115

[12] Duek E. A. R., De Paoli M. A., Mastragostino M.: An electrochromic device based on polyaniline and prussian blue. Advanced Materials, 4, 287-291 (1992). DOI: $10.1002 / \mathrm{adma} .19920040410$

[13] Yang Y., Hao Y., Yuan J., Niu L., Xia F.: In situ preparation of caterpillar-like polyaniline/carbon nanotube hybrids with core shell structure for high performance supercapacitors. Carbon, 78, 279-287 (2014).

DOI: $10.1016 / \mathrm{j}$. carbon.2014.07.004

[14] Chen J-T., Hsu C-S.: Conjugated polymer nanostructures for organic solar cell applications. Polymer Chemistry, 2, 2707-2722 (2011). DOI: $10.1039 / \mathrm{c} 1$ py00275a

[15] Sathiyanarayanan S., Muthkrishnan S., Venkatachari G.: Corrosion protection of steel by polyaniline blended coating. Electrochimica Acta, 51, 6313-6319 (2006). DOI: 10.1016/j.electacta.2006.04.015 
[16] Apodaca D. C., Pernites R. B., Ponnapati R., Del Mundo F. R., Advincula R. C.: Electropolymerized molecularly imprinted polymer film: EIS sensing of bisphenol A. Macromolecules, 44, 6669-6682 (2011). DOI: $10.1021 / \mathrm{ma} 2010525$

[17] Chen M-C., Sun Y-C., Chen Y-H.: Electrically conductive nanofibers with highly oriented structures and their potential application in skeletal muscle tissue engineering. Acta Biomaterialia, 9, 5562-5572 (2013). DOI: $10.1016 /$ j.actbio.2012.10.024

[18] Martin C. R., Parthasarathy R., Menon V.: Template synthesis of electronically conductive polymers - A new route for achieving higher electronic conductivities. Synthetic Metals, 55, 1165-1170 (1993).

DOI: 10.1016/0379-6779(93)90218-1

[19] Parthasarathy R. V., Martin C. R.: Template-synthesized polyaniline microtubules. Chemistry of Materials, 6 , 1627-1632 (1994).

DOI: $10.1021 / \mathrm{cm} 00046 \mathrm{a} 011$

[20] Marquez M., Patel K., Carswell A. D. W., Schmidtke D. W., Grady B. P.: Synthesis of nanometer-scale polymeric structures on surfaces from template assisted admicellar polymerization: A comparative study with protein adsorption. Langmuir, 22, 8010-8016 (2006). DOI: $10.1021 / \mathrm{la} 061108 \mathrm{~g}$

[21] Marquez M., Kim S., Jung J., Truong N., Teeters D., Grady B. P.: Factors affecting the synthesis of polymeric nanostructures from template assisted admicellar polymerization. Langmuir, 23, 10008-10019 (2007). DOI: 10.1021/la7011809

[22] Wu J., Sun Y., Xu W., Zhang Q.: Investigating thermoelectric properties of doped polyaniline nanowires. Synthetic Metals, 189, 177-182 (2014).

DOI: $10.1016 /$ j.synthmet.2014.01.007

[23] Khdary N. H., Abdesalam M. E., El Enany G.: Mesoporous polyaniline films for high performance supercapacitors. Journal of the Electrochemical Society, 161, G63-G68 (2014). DOI: $10.1149 / 2.0441409$ jes

[24] Maiz J., Zhao W., Gu Y., Lawrence J., Arbe A., Alegría A., Emrick T., Colmenero J., Russell T. P., Mijangos C.: Dynamic study of polystyrene-block-poly(4-vinylpyridine) copolymer in bulk and confined in cylindrical nanopores. Polymer, 55, 4057-4066 (2014). DOI: $10.1016 /$ j.polymer.2014.05.042

[25] Serghei A., Chen D., Lee D. H., Russell T. P.: Segmental dynamics of polymers during capillary flow into nanopores. Soft Matter, 6, 1111-1113 (2010).

DOI: $10.1039 / \mathrm{b} 921757 \mathrm{f}$

[26] Blaszczyk-Lezak I., Hernández M., Mijangos C.: One dimensional PMMA nanofibers from AAO templates. Evidence of confinement effects by dielectric and Raman analysis. Macromolecules, 46, 4995-5002 (2013). DOI: $10.1021 / \mathrm{ma} 400173 \mathrm{q}$
[27] Martin J., Mijangos C., Sanz A., Ezquerra T. A., Nogales A.: Segmental dynamics of semicrystalline poly(vinylidene fluoride) nanorods. Macromolecules, 42, 53955401 (2009). DOI: $10.1021 / \mathrm{ma900754 \textrm {v }}$

[28] Suzuki Y., Duran H., Steinhart M., Butt H. J., Floudas G.: Homogeneous crystallization and local dynamics of poly(ethylene oxide) (PEO) confined to nanoporous alumina. Soft Matter, 9, 2621-2628 (2013).

DOI: $10.1039 / \mathrm{c} 2 \mathrm{sm} 27618 \mathrm{f}$

[29] Kremer F., Różański S. A.: The dielectric properties of semiconducting disordered materials. in 'Broadband dielectric spectroscopy’ (eds.: Kremer F., Schönhals A.) Springer, Berlin, 475-494 (2003).

DOI: $10.1007 / 978-3-642-56120-7 \quad 12$

[30] Maiz J., Sacristan J., Mijangos C.: Probing the presence and distribution of single-wall carbon nanotubes in polyvinylidene difluoride 1D nanocomposites by confocal Raman spectroscopy. Chemical Physics Letters, 484, 290-294 (2010).

DOI: $10.1016 /$ j.cplett.2009.11.063

[31] Zhang J., Liu C., Shi G.: Raman spectroscopic study on the structural changes of polyaniline during heating and cooling processes. Journal of Applied Polymer Science, 96, 732-739 (2005).

DOI: 10.1002/app.21520

[32] Salvatierra R. V., Moura L. G., Oliveira M. M., Pimenta M. A., Zarbin A. J. G.: Resonant Raman spectroscopy and spectroelectrochemistry characterization of carbon nanotubes/polyaniline thin film obtained through interfacial polymerization. Journal of Raman Spectroscopy, 43, 1094-1100 (2012). DOI: $10.1002 / j r s .3144$

[33] Stejskal J., Exnerová M., Moravková Z., Trchová M., Hromadková J., Prokeš J.: Oxidative stability of polyaniline. Polymer Degradation and Stability, 97, 10261033 (2012).

DOI: 10.1016/j.polymdegradstab.2012.03.006

[34] Islam R., Chan-Yu-King R., Brun J-F., Gors C., Addad A., Depriester M., Hadj-Sahraoui A., Roussel F.: Transport and thermoelectric properties of polyaniline/reduced graphene oxide nanocomposites. Nanotechnology, 25, 475705/1-475705/9 (2014).

DOI: $10.1088 / 0957-4484 / 25 / 47 / 475705$

[35] Bower D. I.: Infrared dichroism, polarized fluorescence and Raman spectroscopy. in 'Structure and properties of oriented polymers' (ed.: Ward I. M.) Springer, Dordrecht, 181-233 (1997).

DOI: $10.1007 / 978-94-011-5844-24$

[36] Liem H-M., Etchegoin P., Whitehead K-S., Bradley D. D. C.: Raman anisotropy measurements: An effective probe of molecular orientation in conjugated polymer thin films. Advanced Functional Materials, 13, 66-72 (2003).

DOI: $10.1002 / \mathrm{adfm} .200390008$ 
[37] Calleja R. D., Matveeva E. S., Parkhutik V. P.: Electric relaxation in chemically synthesized polyaniline: Study using electric modulus formalism. Journal of NonCrystalline Solids, 180, 260-265 (1995).

DOI: 10.1016/0022-3093(94)00470-6

[38] Moliton A., Hiorns R. C.: Review of electronic and optical properties of semiconducting $\pi$-conjugated polymers: Applications in optoelectronics. Polymer International, 53, 1397-1412 (2004).

DOI: $10.1002 /$ pi.1587

[39] Zhang X., Zhu J., Haldolaarachchige N., Ryu J., Young D. P., Wei S. Y., Guo Z.: Synthetic process engineered polyaniline nanostructures with tunable morphology and physical properties. Polymer, 53, 2109-2120 (2012). DOI: 10.1016/j.polymer.2012.02.042

[40] Prashantha K., Lee J. H.: Positive temperature coefficient characteristics of multi-walled carbon nanotube filled polyvinylidene fluoride nanocomposites. Journal of Macromolecular Science Part A: Pure and Applied Chemistry, 48, 737-741 (2011). DOI: $10.1080 / 10601325.2011 .596056$

[41] Zhang C., Zhu J., Ouyang M., Ma C., Sumita M.: Conductive network formation and electrical properties of poly(vinylidene fluoride)/multiwalled carbon nanotube composites: Percolation and dynamic percolation. Journal of Applied Polymer Science, 114, 1405-1411 (2009). DOI: $10.1002 / a p p .30729$

[42] Almasri A., Ounaies Z., Kim Y. S., Grunlan J.: Characterization of solution-processed double-walled carbon nanotube/poly(vinylidene fluoride) nanocomposites. Macromolecular Materials and Engineering, 293, 123 131 (2008).

DOI: $10.1002 /$ mame.200700229

[43] Li Q., Xue Q., Zheng Q., Hao L., Gao X.: Large dielectric constant of the chemically purified carbon nanotube/polymer composites. Materials Letters, 62, 42294231 (2008).

DOI: $10.1016 /$ j.matlet.2008.06.047

[44] Zhao Z., Zheng W., Yu W., Long B.: Electrical conductivity of poly(vinylidene fluoride)/carbon nanotube composites with a spherical substructure. Carbon, 47, 2118-2120 (2009).

DOI: $10.1016 /$ j.carbon.2009.03.043
[45] Vukićević R., Vukovic I., Stoyanov H., Korwitz A., Pospiech D., Kofod G., Loos K., ten Brinke G., Beuermann S.: Poly(vinylidene fluoride)-functionalized single-walled carbon nanotubes for the preparation of composites with improved conductivity. Polymer Chemistry, 3, 2261-2265 (2012).

DOI: $10.1039 / \mathrm{c} 2$ py20166f

[46] Ansón-Casaos A., González-Domínguez J. M., DiezPascual A. M., Gómez-Fatou M. A., Martínez M. T.: Choosing the chemical route for carbon nanotube integration in poly(vinylidene fluoride). Journal of Physical Chemistry C, 116, 16217-16225 (2012). DOI: $10.1021 / j p 302212 \mathrm{~m}$

[47] Yu S., Zheng W., Yu W., Zhang Y., Jiang Q., Zhao Z.: Formation mechanism of $\beta$-phase in PVDF/CNT composite prepared by the sonication method. Macromolecules, 42, 8870-8874 (2009).

DOI: $10.1021 / \mathrm{ma901765j}$

[48] Sharma M., Madras G., Bose S.: Cooperativity and structural relaxations in PVDF/PMMA blends in the presence of MWNTs: An assessment through SAXS and dielectric spectroscopy. Macromolecules, 47, 13921402 (2014). DOI: $10.1021 / \mathrm{ma} 4023718$

[49] Lonjon A., Demont P., Dantras E., Lacabanne C.: Mechanical improvement of $\mathrm{P}(\mathrm{VDF}-\mathrm{TrFE}) /$ nickel nanowires conductive nanocomposites: Influence of particles aspect ratio. Journal of Non-Crystalline Solids, 358, 236-240 (2012). DOI: 10.1016/j.jnoncrysol.2011.09.019

[50] Li Q., Xue Q. Z., Gao X. L., Zheng Q. B.: Temperature dependence of the electrical properties of the carbon nanotube/polymer composites. Express Polymer Letters, 3, 769-777 (2009).

DOI: $10.3144 /$ expresspolymlett.2009.95

[51] Tao F., Bonnaud L., Auhl D., Struth B., Dubois P., Bailly C.: Influence of shear-induced crystallization on the electrical conductivity of high density polyethylene carbon nanotube nanocomposites. Polymer, 53, 59095916 (2012).

DOI: $10.1016 /$ j.polymer.2012.10.026

[52] Jiang S. L., Yu Y., Xie J. J., Wang L. P., Zeng Y. K., Fu M., Li T.: Positive temperature coefficient properties of multiwall carbon nanotubes/poly(vinylidene fluoride) nanocomposites. Journal of Applied Polymer Science, 116, 838-842 (2010). DOI: 10.1002/app.31569 